\title{
उMR
}

\section{One missense mutation in exon 2 of the PAX5 gene in Iran}

S. Yazdanparast ${ }^{1}$, S.R. Khatami ${ }^{1}$, H. Galehdari ${ }^{1}$ and K. Jaseb ${ }^{2}$

1'Department of Genetics, Faculty of Sciences, Shahid Chamran University of Ahvaz, Ahvaz, Iran

${ }^{2}$ Shafa Hospital and Research Center of Thalassemia and Hemoglobinopathy,

Jundishapur University of Medical Sciences, Ahvaz, Iran

Corresponding author: S.R. Khatami

E-mail: srkhatami@yahoo.com

Genet. Mol. Res. 14 (4): 17768-17775 (2015)

Received August 15, 2015

Accepted October 1, 2015

Published December 22, 2015

DOI http://dx.doi.org/10.4238/2015.December.22.1

ABSTRACT. The PAX5 gene, which encodes the B-cell specific activator protein, is one of the most important factors in determination of B-cell development. This gene is the main target of somatic mutations in acute $B$ lymphoblastic leukemia (B-ALL). For example, point mutations, deletions, as well as other gene rearrangements may lead to several forms of B-cell malignancy. In this study, we obtained 50 blood samples from patients diagnosed with ALL, and screened for PAX5 mutations using sequencing in exons 1, 2 and 3. We found a heterozygous germline variant, c.113G>A (p.Arg38His), which affects the paired domain of PAX5. It seems that this mutation is pathogenic, but is recessive. Our findings suggest that this mutation in a single allele of the PAX5 gene is not sufficient to cause disease, and it is possible that other alleles are also involved in the onset of B-ALL.

Key words: Acute lymphoblastic leukemia; PAX5; Missense mutation; Exon 2; Iran 


\section{INTRODUCTION}

Leukemia is a group of disorders with heterogeneous characters, which leads to neoplastic transformation during differentiation of blood cell precursors in the bone marrow. The most common leukemic cancer is acute lymphoblastic leukemia, which comprises $75 \%$ of all leukemia cases (Pahloosye et al., 2011). B-lineage acute lymphoblastic leukemia (B-ALL), which originates from an early B-cell progenitor, is a common subtype of acute leukemia in children (Sadakane et al., 2007). At the genetic level, B-ALL is determined by a relatively small series of frequent chromosomal alterations, from aneuploidy to chromosomal rearrangements (Campos-Sanchez et al., 2011). The most frequently mutated genetic targets in human lymphoid leukemias are transcription factors, which are essential for blood cell development (O'Neil and Look, 2007). During the transition from common lymphoid progenitors (CLPs) to pro-B cells, commitment to the lineage is dependent on the action of transcription factors such as E2A, EBF1 and PAX5 (Nutt and Kee, 2007). PAX5 expression in the B-lymphocyte lineage is remarkably stable from the pro-B cell stage until its downregulation in plasma cells (Enver, 1999; Cobaleda et al., 2007; Nutt and Kee, 2007). B cells lacking the transcription factor PAX5 cannot complete all the stages of their differentiation. PAX5 is a paired-domain transcription factor that binds to DNA via the N-terminus, and can both positively and negatively regulate transcription (Enver, 1999; Nutt and Kee, 2007). Analysis of gene expression of wild-type and PAX5 knock-out pro-B cells revealed that 110 and 170 genes are repressed and activated, respectively, by PAX5 and that these genes encode essential proteins that participate in several important cell process. PAX5 regulates expression of these genes through induction or elimination of active chromatins (Revilla-i-Domingo et al., 2012). Importantly, PAX5 has also been associated with human B-cell tumors. Frequent inactivation of one of the two PAX5 alleles identified it as a haploinsufficient tumor suppressor gene in B-ALL. Moreover, chromosomal translocation in PAX5 induces its participation in the development of a subset of B-ALL and non-Hodgkin lymphomas (McManus et al., 2011). Furthermore, direct involvement of PAX5 in the development of B-ALLs has also been investigated (Campos-Sanchez et al., 2011). Its alteration, including deletions, point mutations, and amplifications, contributes to approximately $30 \%$ of B-ALL cases, and chromosomal rearrangements occur in 2-3\% of the cases (Campos-Sanchez et al., 2011, Fortscheggeret al., 2014). Genomic analysis of leukemic cells from pediatric ALL patients by Mullighan et al. (2007) showed several changes such as point mutations, deletions, and structural rearrangements in the genes which encodes regulators for $B$ lymphocyte development. Among those, PAX5 was the most mutated somatic gene in children affected with B-ALL (31.7\%). Familiades et al. (2009) examined adult B-ALL patients, and demonstrated that PAX5 is mutated in $34 \%$ of adult B-ALL. These observations suggest that alteration of PAX5 function may contribute to leukemogenesis of B-lineage ALL via improper regulation of early B-cell differentiation (Sadakane et al., 2007). The human PAX5 gene, which encode the protein PAX5, is situated at the 9p13 locus, and composed of 10 exons (Downing and Mullighan, 2006; Arseneau et al., 2009). The coding regions length of the exons is between 46 bp to $198 \mathrm{bp}$. In the present study, the prevalence of probable mutations among B-ALL patients in Khuzestan province of Iran was investigated in exons 1, 2 and 3 of PAX5 gene.

\section{MATERIAL AND METHODS}

The present study was carried out in 50 patients with B-Lineage ALL (31 males, 19 females), who were referred to Shafa hospital in Ahvaz between November 2013 and February 2014. The age of these patients ranged between 1 to 27 years with a mean of 9.4 years. Initial diagnosis of 
ALL was based on clinical phenotypes. After obtaining information of the patients, peripheral blood $(5 \mathrm{~mL})$ was collected into $0.5 \mathrm{M}$ EDTA solution and kept at $-70^{\circ} \mathrm{C}$. Genomic DNA was extracted from white blood cells via the DNA extraction kit from Gen Fanavaran. The forward and reverse primers for exons 1,2 , and 3 on the PAX5 gene were designed with the following sequences: $1 \mathrm{~F}$ (5'-attattccgacctgtgagcg-3') and 1R (5'-gtgtcccggagttgcac-3'), 2F (5'agcggtgcttctcctatgtg-3') and $2 R$ (5'-acagctgctgggtcatgttt-3'), 3F (5'-tagcccgttattttgttgcc-3') and 3R (5'-atccctccaaatccccaac-3'). PCR reactions were performed in a total volume of $25 \mu \mathrm{L}$ with the following cycling parameters: $94^{\circ} \mathrm{C}$ for $5 \mathrm{~min}$; 35 cycles of $94^{\circ} \mathrm{C}$ for $30 \mathrm{~s}, 60^{\circ} \mathrm{C}$ for $45 \mathrm{~s}$ and $72^{\circ} \mathrm{C}$ for $30 \mathrm{~s}$, followed by one cycle of $72^{\circ} \mathrm{C}$ for $3 \mathrm{~min}$. The PCR products were run on $1.5 \%$ agarose gels to verify the fidelity of PCR reactions. Direct sequencing of exons 1,2 and 3 was carried out with the big dye terminator cycle sequencing ready reaction kit (Applied Biosystems) on an ABI prism 3500 automated genetic analyzer (Applied Biosystems). Sequencing reactions were accomplished by the same primers that were used for exons amplification. Sequencing analyses were performed using Chromas, and the results were compared with the reported gene sequences. Estimation of the functional outcomes of mutations on protein structure was accomplished via Predict SNP and Mutation Taster.

\section{RESULTS}

The occurrence of PAX5 mutations were examined in a group of $50 \mathrm{~B}-\mathrm{ALL}$ patients. Direct sequencing analysis of the patients, after comparison with the PAX5 reference sequence, revealed a heterozygous missense germline variant CGC > CAC substitution in exon 2. This mutation was found in codon 38 of the PAX5 gene in one of the patients, an 18-years old male with B-ALL. At the protein level, this mutation causes an amino acid substitution from arginine to histidine. Sequencing of the PAX5 gene in the family members of patient revealed that his mother also was heterozygous for the mentioned mutation but his father and brother did not show any mutations (Figure 1).

A

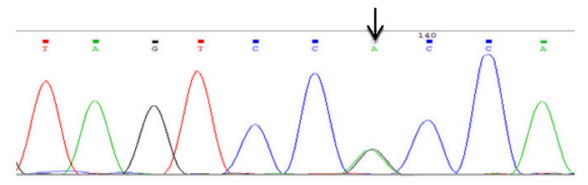

B

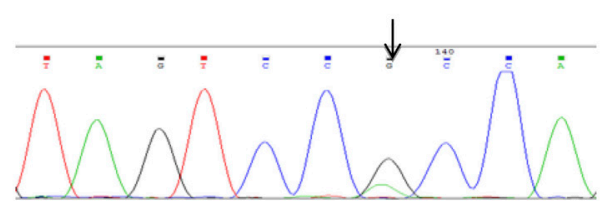

C

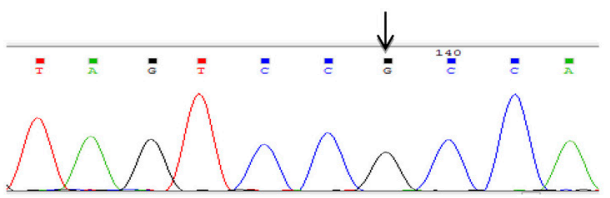

Figure 1. Sequencing results from A. the child with ALL, who was heterozygous for the mentioned mutation; B. the mother of the affected boy, who was heterozygous for the mentioned mutation; and $\mathbf{C}$. the father of the affected boy, showing a normal sequence (result of the sequencing of the patient's brother was similar as his father) (sequencing with reverse primer). 
The missense mutation (p.R38H) declared in this patient, was analyzed by Predict SNP and Mutation Taster. Accordingly, Predict SNP suggests that the missense mutation has a diseasecausing probability of $87 \%$. The $\mathrm{R} 38 \mathrm{H}$ variant was also forecasted as disease causing by Mutation Taster with a $99 \%$ probability.

\section{DISCUSSION}

One of the malignant disorders of immature lymphoid cells that affect both children and adults is acute lymphoblastic leukemia (Pui et al., 2008). In children 1 to 4 years of age, the incidence of this disease is 10 times higher than that in 20 to 24 year old adults (Rodriguez-Abreu et al., 2007). Out of the 50 subjects that we recruited for the study, 10 patients were between 1 and 5 years of age, 18 patients were between 5 and 10 years old, 11 patients were between 10 and 15 years old, 10 patients were between 15 and 20 years old, and one patient was 20-27 years old. Worldwide incidence for this disease is projected to be 1-4.75 per 100,000 people (Redaelli et al., 2005). Annual incidence of leukemia in some parts of Iran is approximately 3.3 to 5.6 per 100,000 people (Dastgiri et al., 2011). It is believed that the etiology of ALL is multifactorial, and is likely contributed by interactions between environmental and genetic variables (Ellinghaus et al., 2012). Therefore, it is important to examine the genetic components in different populations due to differences in genetic background and other factors that are specific for a certain population. Submicroscopic genetic alterations such as changes in B-lymphoid transcription factors have been recognized in ALL as important determinants of leukemia (Holmfeldt et al., 2013). The most common alterations would be in transcription regulators of B-lymphoid developments such as PAX5, EBF1, and IKZF1, which accounts for more than $60 \%$ of the B-ALL cases (Inaba et al., 2013). PAX5 is the main target of somatic mutations in B-ALL, and mutations in this gene is estimated to account for one-third of B-ALL patients (Mullighan et al., 2009; Mullighan, 2012), indicating its importance in B-ALL pathophysiology. In spite of studies on somatic mutations in PAX5, few studies were conducted on the inherited mutations of the PAX5 gene (Shah et al., 2013). In the present work, probable mutations in exons 1, 2 and 3of the PAX5 gene in patients with B-ALL were studied in the Khuzestan province of Iran. For this screening, following PCR, the exons were directly sequenced, and one heterozygous germline variant, c .113G > A encoding ( $p$.Arg38His), was found in one of the patients. It was a missense mutation $G>A(R 38 H)$ located in exon 2 of the PAX5 gene. The known missense variant of $p$.Arg38Gly, is located in a conserved residue in the paired domain of PAX5 (Figure 2).

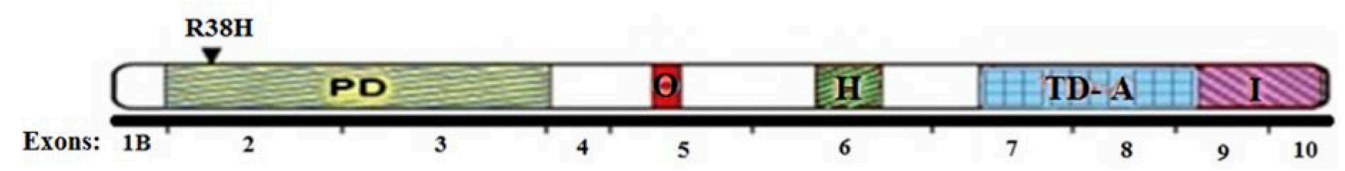

Figure 2. Schematic of exons that encode various domains of PAX5, and the mutation site which was found. In the domains, we can see the paired domain, octapeptide domain, homeodomain, transactivating domain, activating domain, and inhibitory domain, which are denoted with PD, O, H, TD, A, and I, respectively.

In the past few years, the $\mathrm{R} 38 \mathrm{H}$ variant has been identified in several studies. Bernardi et al. (2013) found this variant in an Italian patient who suffered from B-ALL and Alagille syndrome. They identified damaging mutations in genes such as PAX5 (R38H) and NOTCH1 (K1821N) which may be strongly related to the observed disease (Bernardi et al., 2013). In addition, Santoro et al. 
(2009) also recognized the $\mathrm{R} 38 \mathrm{H}$ variant in B-ALL patients. They declared that point mutations of the PAX5 gene along with other changes such as alternative splicing of PAX5, may be involved in the pathogenesis of B-ALL in both adults and children (Santoro et al., 2009). Furthermore, Miller et al. (2008) examined the PAX5 gene in murine models and proposed that the $\mathrm{R} 38 \mathrm{H}$ variant causes defective binding of PAX5 to DNA. These studies all suggest that PAX5 contributes to leukemogenesis.

According to accomplished software analyses by Mutation Taster, location of the reported mutation, codon 38 , is highly conserved between species, and substitution of arginine by histidine leads to the mutated form of the protein (Table 1). In addition, as illustrated below, even regions surrounding codon 38 show highly conserved sequences, which further suggests that in this area may have profound effects on the protein structure and function.

Table 1. Conservation of codon 38 of the PAX5 gene in different species.

\begin{tabular}{|c|c|c|c|}
\hline Species & Gene & Amino acids & Alignment \\
\hline Human & ENSG00000196092 & 38 & FVNGRPLPDVVRQRIVELAHQGVR \\
\hline Mutated & & 38 & FVNGRPLPDVVHQRIVELAHQGV \\
\hline P. troglodytes & ENSPTRG00000020936 & 38 & F VNGRPLPD V VRQRIVELAHQG V \\
\hline M. mulatta & ENSMMUG00000019639 & 38 & FVNGRPLPD V VRQRIVELAHQG V \\
\hline F. catus & ENSFCAG00000001628 & 38 & FVNGRPLPD V VRQRIVELAHQG V \\
\hline M. musculus & ENSMUSG00000014030 & 38 & FVNGRPLPDVVRQRIVELAHQG V \\
\hline G. gallus & ENSGALG00000016431 & 38 & FVNGRPLPD V VRQRIVELAHQG V \\
\hline T. rubripes & ENSTRUG00000003227 & 38 & F VNGRPLPD V VRQRIVELAHQG V \\
\hline D. rerio & ENSDARG00000037383 & 38 & FVNGRPLPD V VRQRIVELAHQG V \\
\hline X. tropicalis & ENSXETG00000006547 & 38 & FVNGRPLPD V VRQRIVELAHQG V \\
\hline
\end{tabular}

Arginine is a positively charged amino acid. Analysis of proteins which interface with DNA indicates that these proteins are highly enriched in positive charges and are almost devoid of negative charges. On the DNA side, negatively charged phosphate residues represent approximately $43 \%$ of the surface area that is in contact with the proteins (Nadassy., 1999). Histidine is an ionizable amino acid with an ionization constant, or pKa value, of approximately 6.5 (Liao et al., 2013). Since arginine has a positive charge and histidine has a nearly neutral charge, substitution between the two amino acids may significantly affect protein affinity to DNA.

Analysis of the PAX5 mutations with respect to their locations within the DNA-binding domains suggested that these alterations may change or eliminate its binding to the DNA (Figure 3 ).

A

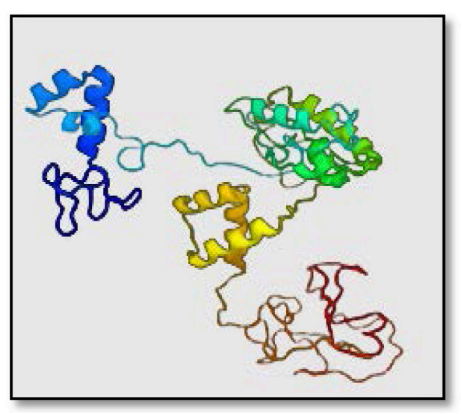

Figure 3. Three-dimensional structure of the PAX5 protein. A. Normal protein. B. Mutant protein.
B

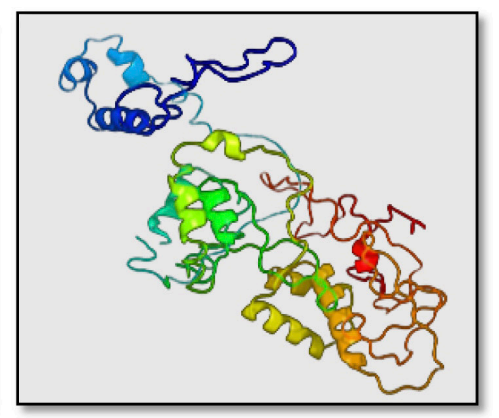


The function of a protein is intimately linked to its structural properties. Because of the role of PAX5 in many cellular processes such as transcription control, receptor signaling, adhesion, migration, and immune function, change in protein structure may cause defects in many of these processes. The location of the $\mathrm{R} 38 \mathrm{H}$ variant is in the paired domain of PAX5. The paired domain is a conserved DNA-binding motif, and can act as transcriptional repressors or activators. It is possible that alterations to the paired domain may affect its binding to DNA. However, to determine the exact effect of the $\mathrm{R} 38 \mathrm{H}$ variant, further functional analyses are required.

Here we present separate images of the region of exons 1, 2, and 3 that make up the paired domain PAX5, and show the changes in structure as a result of the mutation (Figure 4). According to prediction of "Predict SNP" and "Mutation Taster", the probability of this mutation in causing disease is high.

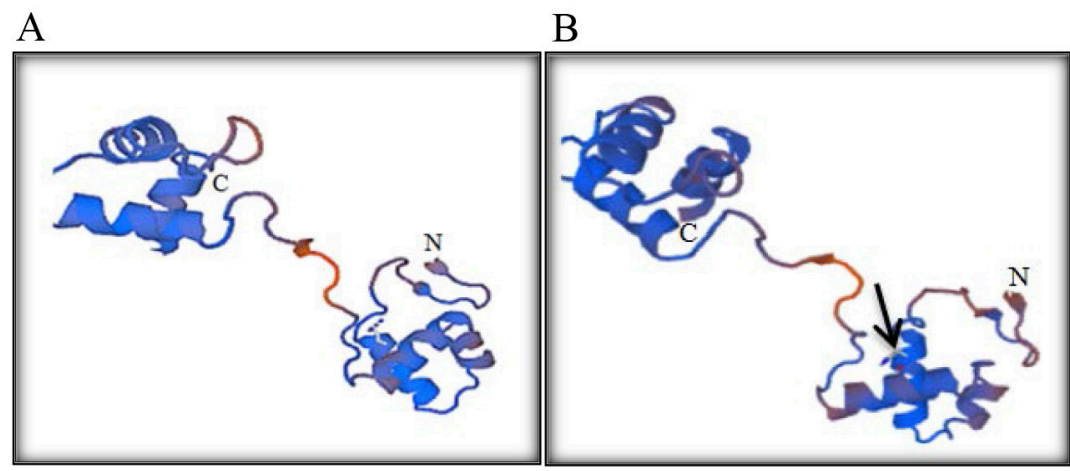

Figure 4. Picture of the paired domain of PAX5 and location of its mutation. A. Normal, B. mutant. Arrow indicates the position of the mutation.

After observing the mutation in our patient, sequencing of the PAX5 gene in exon 2 have been performed on all of the family members, including the father, mother, and brother of the patient, who didn't show any clinical feature of the disease. Based on the sequencing results, the mutation was present in the patient's mother in the heterozygous form, but not in either the father or the brother. This indicates that the boy inherited the allele from his mother. Interestingly, despite having the mutation, the mother is healthy, which suggests that the mutation is of a recessive origin. Therefore, the other allele on the mother's gene must be normal to achieve proper gene function. As recessive diseases require changes in both copies of the gene, the mutated allele from the patient was inherited from the mother as a germline mutation. However, since this defective allele is not sufficient to cause disease, it is possible that genetic defect on another allele of this gene may have occurred. As a result, normal gene function is lost. The origin of the other mutation is unknown; however, the new change in the other allele may be a somatic one. Therefore, while we have found one heritable mutation, it seems that more molecular and cytogenetic analyses are needed in order to explore every probable change in the alleles of PAX5 gene.

\section{Conflicts of interest}

The authors declare no conflict of interests. 


\section{ACKNOWLEDGMENTS}

The authors would like to thank the patient families involved in this study and the Shafa hospital of Ahvaz for providing leukemia samples. This study was supported in part by grant \#867615 from the Shahid Chamran University.

\section{REFERENCES}

Arseneau JR, Laflamme M, Lewis SM, Maïcas E, et al. (2009). Multiple isoforms of PAX5 are expressed in both lymphomas and normal B-cells. Br. J. Haematol. 147: 328-338.

Bernardi S, Tononi P, Vargas SM, Bassi G, et al. (2013). Genomic analysis of notch mutations in a case of Alagille syndrome with acute lymphoblastic leukemia. Blood 122: 4992.

Campos-Sanchez E, Toboso-Navasa A, Romero-Camarero I, Barajas-Diego M, et al. (2011). Acute lymphoblastic leukemia and developmental biology: a crucial interrelationship. Cell Cycle 10: 3473-3486.

Cobaleda C, Schebesta A, Delogu A and Busslinger M (2007). PAX5: the guardian of B cell identity and function. Nat. Immunol. 8: $463-470$.

Dastgiri S, Fozounkhah S, Shokrgozar S, Taghavini M, et al. (2011). Incidence of Leukemia in the Northwest of Iran. Health Promot. Perspect. 1: 50-53.

Downing JR and Mullighan CG (2006). Tumor-specific genetic lesions and their influence on therapy in pediatric acute lymphoblastic leukemia. Hematol. Am. Soc. Hematol. Educ. Program. 2006: 118-122.

Ellinghaus E, Stanulla M, Richter G, Ellinghaus D, et al. (2012). Identification of germline susceptibility loci in ETV6-RUNX1rearranged childhood acute lymphoblastic leukemia. Leukemia 26: 902-909.

Enver T (1999). B-cell commitment: Pax5 is the deciding factor. Curr. Biol. 9: R933-R935.

Familiades J, Bousquet M, Lafage-Pochitaloff M, Béné MC, et al. (2009). PAX5 mutations occur frequently in adult B-cell progenitor acute lymphoblastic leukemia and PAX5 haploinsufficiency is associated with BCR-ABL1 and TCF3-PBX1 fusion genes: a GRAALL study. Leukemia 23: 1989-1998.

Fortschegger K, Anderl, S, Denk D and StrehI S (2014). Functional heterogeneity of PAX5 chimeras reveals Insight for leukemia development. Mol. Cancer Res. 12: 595-606.

Holmfeldt L, Wei L, Diaz-Flores E, Walsh M, et al. (2013). The genomic landscape of hypodiploid acute lymphoblastic leukemia. Nat. Genet. 45: 242-252.

Inaba H, Greaves M and Mullighan CG (2013). Acute lymphoblastic leukemia. Lancet 381: 1-27.

Liao SM, Du QS, Meng JZ, Pang ZW, et al. (2013). The multiple roles of histidine in protein interactions. Chem. Cent. J. 7: 44.

McManus S, Ebert A, Salvagiotto G, Medvedovic J, et al. (2011). The transcription factor Pax5 regulates its target genes by recruiting chromatin-modifying proteins in committed B cells. EMBO J. 30: 2388-2404.

Miller CS, Mullighan CG, Su X, Ma J, et al. (2008). Pax5 haploinsufficiency cooperates with BCR-ABL1 to induce acute lymphoblastic leukemia. Blood 112: Abstract 293.

Mullighan CG (2012). The molecular genetic makeup of acute lymphoblastic leukemia. Hematol. Am. Soc. Hematol. Educ. Program 2012: 389-396.

Mullighan CG, Goorha S, Radtke I, Miller CB, et al. (2007). Genome-wide analysis of genetic alterations in acute lymphoblastic leukemia. Nature 446: 758-764.

Mullighan GC, Su X, Zhang J, Radtke I, et al. (2009). Deletion of IKZF1 and prognosis in acute lymphoblastic leukemia. N. Engl. J. Med. 360: 470-480.

Nadassy K, Wodak SJ and Janin J (1999). Structural features of protein-nucleic acid recognition sites. Biochemistry 38: 19992017.

Nutt SL and Kee BL (2007). The transcriptional regulation of B cell lineage commitment. Immunity 26: 715-725.

O'Neil J and Look AT (2007). Mechanisms of transcription factor deregulation in lymphoid cell transformation. Oncogene 26: 6838-6849.

Pahloosye A, Hashemi A, Mirmohammadi SJ and Atefi A (2011). Presenting clinical and laboratory data of childhood acute lymphoblastic leukemia. Iran. J. Ped. Hematol. Oncol. 1: 71-77.

Pui CH, Robison LL and Look AT (2008). Acute lymphoblastic leukemia. Lancet 371: 1030-1043.

Redaelli A, Laskin BL, Stephens JM, Botteman MF, et al. (2005). A systematic literature review of the clinical and epidemiological burden of acute lymphoblastic leukaemia (ALL). Eur. J. Cancer Care 4: 53-62.

Revilla-i-Domingo R, Bilic I, Vilagos B, Tagoh H, et al. (2012). The B-cell identity factor Pax5 regulates distinct transcriptional programmes in early and late B lymphopoiesis. EMBO J. 31: 3130-3146. 
Rodriguez-Abreu D, Bordoni A and Zucca E (2007). Epidemiology of hematological malignancies. Ann. Oncol. 18 (Suppl 1): i3-i8.

Sadakane Y, Zaitsu M, Nishi M, Sugita K, et al. (2007). Expression and production of aberrant PAX5 with deletion of exon 8 in B-lineage acute lymphoblastic leukaemia of children. Br. J. Haematol. 136: 297-300.

Santoro A, Bica MG, Dagnino L, Agueli C, et al. (2009). Altered mRNA expression of PAX5 is a common event in acute lymphoblastic leukaemia. Br. J. Haematol. 146: 686-689.

Shah S, Schrader KA, Waanders E, Timms AE, et al. (2013). A recurrent germline PAX5 mutation confers susceptibility to pre-B cell acute lymphoblastic leukemia. Nat. Genet. 45: 1226-1231. 